\title{
QUÍMICA COMBINATÓRIA DE MATERIAIS COM ANÁLISE POR MICRODIFRAÇÃO DE RAIOS X. PRIMEIRA PARTE: FUNDAMENTOS
}

\author{
Najla Maroun Estrada \\ Centro de Pesquisas da Petrobras, CENPES, Gerência de Química, Ilha do Fundão, Quadra 7, Radial 3, \\ 21949-900 Rio de Janeiro - RJ \\ Francisco Manoel dos Santos Garrido*, Marta Eloisa Medeiros e Sérgio de Paula Machado \\ Instituto de Química, Universidade Federal do Rio de Janeiro, Centro de Tecnologia, Bloco A, Ilha do Fundão, \\ 21945-970 Rio de Janeiro - RJ
}

Recebido em 14/6/04; aceito em 5/10/04; publicado na web em 28/2/05

\begin{abstract}
COMBINATORIAL CHEMISTRY OF MATERIALS WITH ANALYSIS BY X-RAY MICRODIFFRACTION. PART I: FUNDAMENTALS: Combinatorial chemistry refers to techniques to rapidly fabricate tens, hundreds or even thousands of different micro samples. The analysis of the large number of samples generated by combinatorial methods requires highly efficient analytical methods. In this case, the challenges are due not only to the large number of samples to be analyzed, but also to the small amount of sample available for analysis. This paper describes the fundamentals of combinatorial chemical methods applied to discover of materials and the development in $\mathrm{x}$-ray diffraction to analyze micro samples.
\end{abstract}

Keywords: combinatorial chemistry; materials chemistry; X-ray diffraction.

\section{INTRODUÇÃO}

Os processos convencionais de descobrimento de novos materiais em áreas importantes como catálise, supercondutores e semicondutores são demorados e caros devido aos inúmeros passos, realizados um de cada vez, necessários para produzir um material novo e otimizado.

A natureza utiliza uma abordagem mais dinâmica para produzir o número imenso de moléculas que acompanham o complexo processo de vida dos organismos. Diferentemente do químico convencional, que sintetiza e caracteriza uma molécula de cada vez, a natureza delineia uma vasta biblioteca combinatória ("library"), a partir de um conjunto de moléculas precursoras ("building blocks") e testa ("screening") os componentes dessa biblioteca em relação às propriedades desejadas. Talvez o exemplo mais importante desta estratégia seja o sistema imunológico, que é capaz de gerar uma imensa variedade de moléculas através de rearranjos genéticos e mutações somáticas, testando-as quanto à afinidade e seletividade a novos antígenos.

O poder do exemplo natural dos processos combinatórios inspirou químicos e bioquímicos a aplicarem esta estratégia a outros problemas, variando desde a descoberta de medicamentos e catalisadores até a ciência dos materiais ${ }^{1-8}$. Esta abordagem foi primeiro aplicada na indústria farmacêutica, onde os altos custos e os tempos longos das pesquisas que utilizam metodologias convencionais forçaram o desenvolvimento de novas abordagens, com base na química combinatória, que tornaram possível um aumento dramático na velocidade do processo de descoberta de novos fármacos ${ }^{5-8}$. $\mathrm{Na}$ área de polímeros, a química combinatória tem sido aplicada tanto para o desenvolvimento de novos e melhores polímeros, como para a descoberta de novos catalisadores de polimerização9. Com relação à catálise heterogênea, onde os sítios ativos existem na superfície exterior e/ou no interior de um material inorgânico sóli-

*e-mail: chico@iq.ufrj.br do, muitas vezes são utilizadas abordagens similares às da área de ciência dos materiais ${ }^{3,5,10,11}$. Para a catálise homogênea, onde os sítios ativos são normalmente um íon metálico estabilizado por um ligante orgânico, têm sido desenvolvidas abordagens específicas ${ }^{10}$.

Em anos recentes, vem se observando um grande aumento nas pesquisas que utilizam a abordagem da química combinatória em vários ramos da química; a publicação de vários artigos de revisão $3,5,7-16$ e a criação de novas revistas ("Molecular Diversity", "Combinatorial Chemistry" e "Journal of Combinatorial Chemistry") confirmam este fato.

A química combinatória é uma tecnologia composta por uma série de técnicas idealizadas para a obtenção de um grande número de compostos ou misturas ("library"), a partir de um conjunto de moléculas precursoras ("building blocks") que são combinadas pelas maneiras mais diversas possíveis; a seguir, os componentes da biblioteca são avaliados com relação às propriedades de interesse, sendo que todas as etapas envolvidas na síntese e avaliação devem ser realizadas da forma mais rápida possível.

Os primeiros trabalhos, com esta abordagem, foram reportados há mais de 15 anos, utilizando processos aplicados na indústria farmacêutica para síntese e varredura ("screening") de uma vasta biblioteca de compostos orgânicos ${ }^{8}$. Em 1995 foi realizado um trabalho na área não farmacêutica, que mostrou que os métodos combinatórios também podiam ser aplicados no estudo de óxidos metálicos complexos, com potencial para utilização como supercondutores ${ }^{4}$.

Por ser uma metodologia de acesso a um grande volume de informações químicas, as tecnologias combinatórias podem proporcionar oportunidades para aquisição de conhecimentos em meses, daquilo que demoraria anos com o uso de metodologias de pesquisa tradicionais ${ }^{5}$. No Gráfico 1 é mostrada a comparação entre a capacidade de produção de amostras com utilização de metodologia convencional e combinatória, ficando evidente a grande vantagem desta última. É importante ressaltar que, em função de suas características, os métodos combinatórios ganham relevância, em relação às outras abordagens científicas, quando se tem um baixo nível de co- 


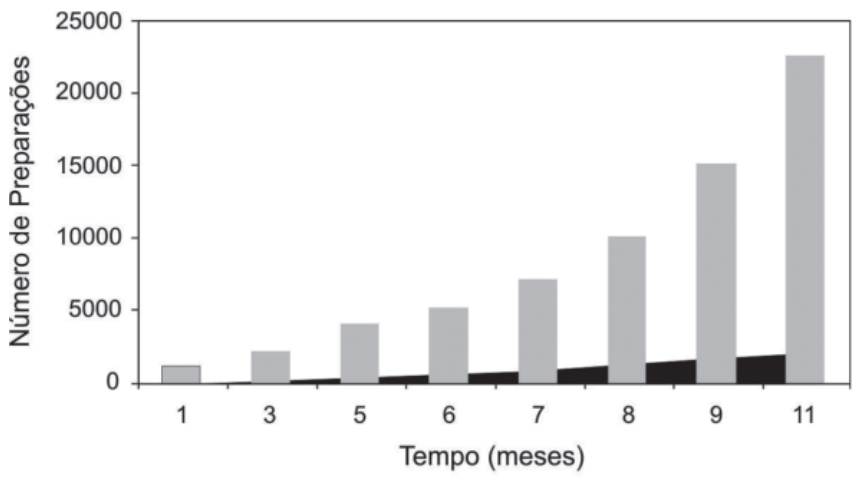

Reatores Convencionais

Reatores Combinatoriais

Gráfico 1. Comparação entre preparações convencionais e combinatórias

nhecimento científico sobre o sistema a ser estudado; caso contrário, abordagens convencionais são mais apropriadas ${ }^{5}$.

Neste artigo serão apresentados os conceitos fundamentais da química combinatória, sendo que algumas metodologias de síntese das bibliotecas serão abordadas de forma mais detalhada. Com relação aos métodos de avaliação, será dispensada atenção especial para o método de microdifração de raios X (MDRX).

\section{O PROCESSO DA QUÍMICA COMBINATÓRIA}

As etapas chaves do processo da química combinatória são:

- definição do objetivo, utilizando a opinião de especialistas, hipóteses e modelagem molecular, quando possível.

- projeto da biblioteca via insumos computacionais, tais como relações propriedade-estrutura e modelagem molecular. Planejamentos de experimentos são utilizados na redução do número de amostras necessárias para alcançar os objetivos definidos. Dados de "screenings" prévios podem ser utilizados como ponto de partida;

- fabricação da biblioteca, envolvendo preparo e/ou processamento de matrizes de dimensão $n$ de amostras físicas; geralmente é necessário se gerar centenas de amostras;

- varredura ("screening") da biblioteca, podendo envolver uso de robótica e sensores que, rápida e automaticamente, analisam a biblioteca de amostras na busca das propriedades desejadas e

- coleta e análise de dados usando bases de dados e, em alguns casos, ferramentas de inteligência artificial.

Um fluxograma mais detalhado do processo combinatório é mostrado na Figura 1. Como pode ser visto nessa figura, a metodologia combinatória compreende uma etapa de "discovery", ou etapa 1, onde um número maciço de amostras é fabricado e analisado, através da medida rápida de uma ou mais propriedades físico-químicas de cada um dos membros da biblioteca, levando à identificação de possíveis candidatos à produção industrial, chamados de "hits". Na etapa 2, uma nova biblioteca é preparada a partir dos "hits" identificados e o ciclo do método combinatório completase. Como produto, é obtida família de compostos ("leads") com as propriedades desejadas. Em seguida, a etapa 2 pode ser repetida uma ou mais vezes, para que os "leads" sejam otimizados pela variação contínua da composição, estrutura e outras propriedades. Numa etapa posterior, de validação, os materiais otimizados são sintetizados em quantidades suficientes para uma caracterização detalhada. Finalmente, é realizada a etapa de aumento de escala ("scale-up"). Em cada uma dessas etapas há envolvimento de um certo número de compostos, podendo ser de até 100.000 na etapa 1 , chegando a apenas um na etapa final ${ }^{8}$.

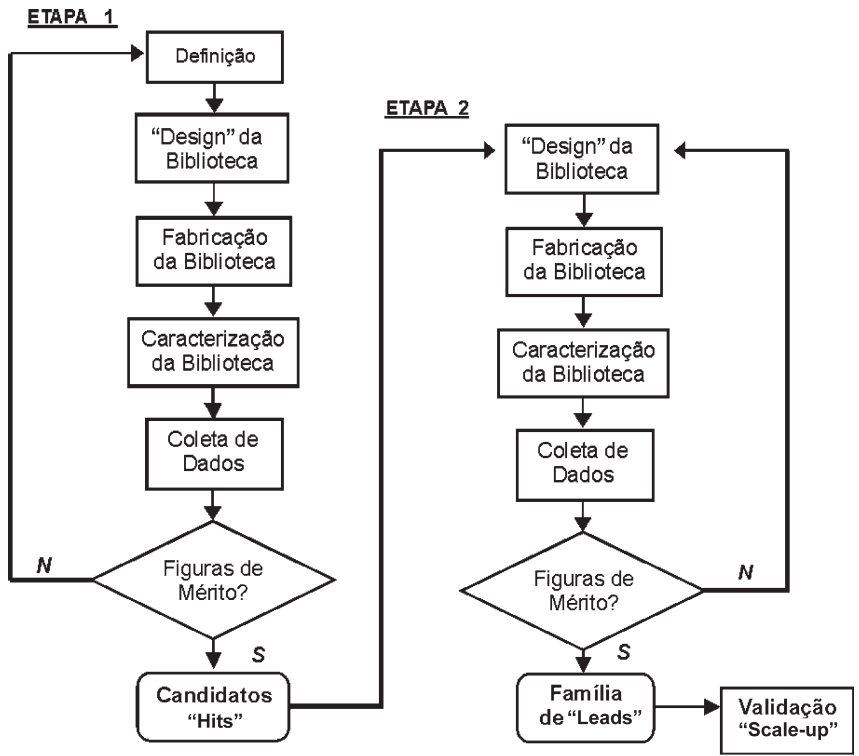

Figura 1. Fluxograma do processo combinatório

Quando aplicadas na descoberta de novos materiais, catalisadores e polímeros, as etapas de planejamento ("design"), síntese e de avaliação das bibliotecas, montadas para a etapa de "discovery", são agrupadas na literatura ${ }^{5,16}$ sob o termo HTE ("High Throughput Experimentation").

\section{PLANEJAMENTO DA BIBLIOTECA}

A aplicação da química combinatória na descoberta de novos materiais e/ou drogas envolve um número muito grande de combinações possíveis de parâmetros relevantes. Mesmo no caso de já se conhecer a estrutura do alvo, há necessidade de estratégias para reduzir o número de materiais a serem preparados, uma vez que as combinações possíveis representam um número muito grande para testes individuais; para resolver este problema geralmente são utilizadas ferramentas estatísticas.

As estratégias mais adequadas para otimizar formulações já existentes são aquelas baseadas nos métodos estocásticos ${ }^{14}-$ métodos matemáticos que envolvem a teoria das probabilidades. Dentre esses métodos, os principais seriam o dos algoritmos genéticos (GA) e as simulações pelos métodos Monte Carlo ${ }^{5,14}$.

\section{METODOLOGIAS DE SÍNTESE}

A etapa de fabricação da biblioteca consiste na síntese de uma multiplicidade de compostos químicos, que serão, a seguir, avaliados. Consideremos como exemplo uma reação de uma molécula precursora de classe A (um ácido de Lewis) com uma molécula precursora de classe B (uma base de Lewis) em um solvente $S$, para gerar um produto $\mathrm{P}$. Combinando $n$ diferentes compostos de classe A com $m$ diferentes compostos de classe $\mathrm{B}$, em $l$ diferentes solventes $\mathrm{S}$, teríamos como resultado um total de $(n \times m \times l)$ diferentes experimentos a serem realizados, no caso de não se utilizar nenhuma estratégia estatística de planejamento experimental. Cabe ressaltar que cada um destes experimentos pode levar a diferentes produtos ou misturas, ou ainda, a diferentes rendimentos de um determinado produto.

Existem quatro estratégias principais de síntese para a produção de um conjunto de amostras ${ }^{5,16-21}$ : síntese paralela, "split and mix", síntese via "thin-film vapor deposition" e a síntese via "ink jet". 


\section{Síntese paralela}

Na síntese paralela cada um dos produtos é gerado individualmente. Esta estratégia pode empregar métodos em fase sólida ou em fase líquida e permite o uso de sistemas robóticos ${ }^{17}$. As sínteses em solução são feitas freqüentemente em uma placa de microtitulação, que é um disco raso cuja superfície superior contém um grande número de cavidades chamadas de poços ("wells"). O conjunto de linhas e colunas de poços permite que os pesquisadores localizem os "building blocks" que foram combinados, proporcionando uma maneira simples de identificar compostos de um poço em particular. A Figura 2 mostra um esquema de síntese paralela para a produção de 96 amidas distintas, a partir de 20 "building blocks" (8 aminas diferentes, colocadas nas linhas e 12 ácidos carboxílicos diferentes, colocados nas colunas). Em sínteses paralelas no estado sólido são utilizados equipamentos robóticos para coletar e misturar os reagentes nos diferentes poços ou vasos miniaturas.

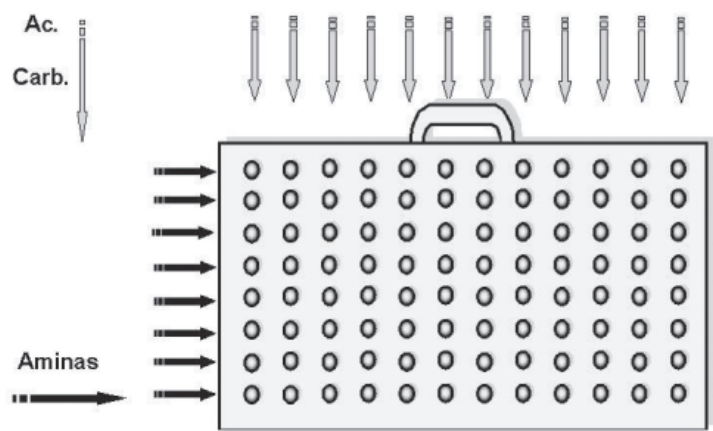

Figura 2. Síntese paralela a partir de soluções

Uma das vantagens da síntese paralela é a identificação das substâncias ativas após o "screening", uma vez que estão em poços diferentes. Entretanto, este método leva a uma desvantagem na etapa de avaliação, pois é difícil encontrar um modo apropriado para testar todos os produtos isolados, utilizando altas velocidades de "screening" ("high troughput screening"). A existência de uma etapa lenta no ciclo combinatório (planejamento, síntese e avaliação) minimiza os benefícios conseguidos. Então, métodos analíticos rápidos estão sendo desenvolvidos por muitos grupos de pesquisa. Baseiam-se no uso de ferramentas analíticas otimizadas, automatizadas e de leituras simultâneas, como a microdifração de raios X, cromatografia e termografia, dentre outras ${ }^{16-21}$.

\section{Síntese "split and mix"}

Essa estratégia só emprega métodos em fase sólida ${ }^{8,19}$. Geralmente está relacionada ao uso de corpos ("beads") de resinas poliméricas como suportes para a síntese de compostos bioativos como, por ex., polipeptídeos. No caso da síntese de materiais inorgânicos, podem ser utilizados corpos de alumina porosa ${ }^{19}$. Em contraste com a síntese paralela, os poços podem conter uma mistura de produtos relacionados reduzindo, dessa forma, o número de vasos e aumentando o número de compostos que podem ser gerados.

Na primeira etapa desta estratégia é realizada a adição de $n$ reagentes ("building blocks") em $n$ vasos de reação, cada um contendo um número determinado de suportes poliméricos, que irão reagir ou ser impregnados com um desses compostos. Depois de todas as reações se completarem, o excesso dos reagentes é eliminado por lavagem. É realizado então o processo de mistura, onde o conteúdo de cada um dos vasos é igualmente repartido entre $n$ vasos novos, a seguir os mesmos $n$ "building blocks" são novamente adicionados, um em cada vaso. Este procedimento é repetido $m$ vezes ( $m$ etapas) de maneira a gerar os $n^{m}$ componentes da biblioteca, sendo que este é, exatamente, o número total de suportes poliméricos usados inicialmente.

Na Figura 3 é apresentado um esquema para a preparação de uma biblioteca com 27 componentes $\left(3^{3}\right)$, onde são utilizados 3 reagentes diferentes e o processo é repetido 3 vezes. Como pode ser visto, na primeira etapa são obtidos três componentes $(3 \mathrm{X} 1=$ 3 ); todos os suportes de um vaso estão impregnados ou reagiram com um dos três reagentes. Depois de sofrerem o processo de mistura, onde o conteúdo de cada um dos vasos é igualmente dividido entre três vasos novos, passa-se para a segunda etapa onde são obtidos 9 componentes ( $3 \times 3=9)$; cada suporte foi impregnado ou reagiu com dois "building blocks". Finalmente, na terceira etapa são obtidos os 27 componentes ( 3 X 3 X $3=27$ ).

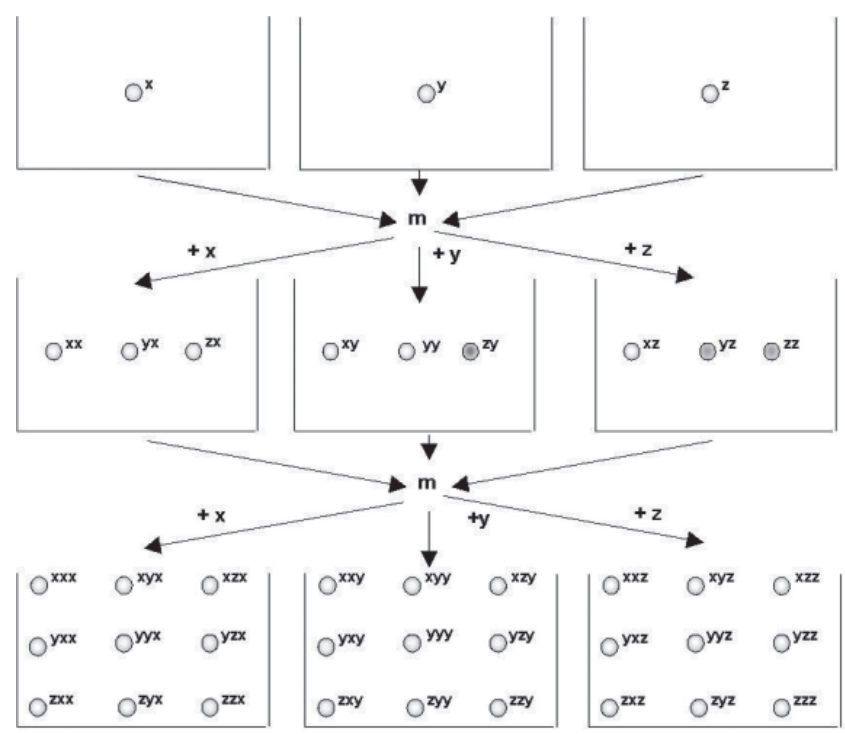

Figura 3. "Split and mix" de uma biblioteca de 27 elementos em três etapas, a partir da combinação de 3 reagentes

Uma vez criada, essa biblioteca precisa ser testada para determinar os compostos ativos. Quando a síntese "split and mix" é usada, surge o problema de identificação dos compostos que apresentaram atividade durante o "screening". Uma maneira de resolver esse problema é o processo de deconvolução ${ }^{8}$. Na Figura 4 é apresentado o processo de deconvolução para o exemplo da Figura 3. Na primeira etapa, os elementos da biblioteca são divididos em $n$ vasos e avaliados quanto à atividade: o vaso ativo é identificado e os demais descartados. Os compostos do vaso ativo são sintetizados novamente em bibliotecas menores e novamente avaliados quanto à atividade. Esse processo de eliminação se repete até que o composto ativo é identificado. Apesar do método de deconvolução ter se mostrado útil, apresenta algumas desvantagens: é um processo que consome bastante tempo e, uma vez identificado o vaso com atividade, não há certeza de que a atividade seja proveniente de um único produto ativo ou resultado da presença de muitos compostos ativos.

Comparando as duas primeiras estratégias de síntese, o método "split and mix" é mais útil quando é necessária a avaliação de um grande número de compostos: biblioteca diversa. Por outro lado, a síntese paralela seria mais aplicada quando algum conhecimento sobre a estrutura do produto alvo está disponível ou quando somente uma otimização do alvo é requerida: biblioteca focada. Finalmente, é necessário enfatizar que a síntese "split and mix" é 


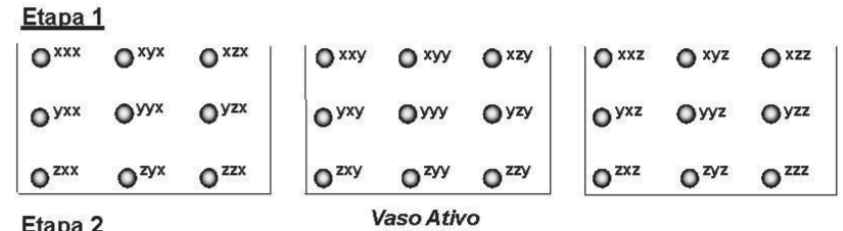

Etapa 2
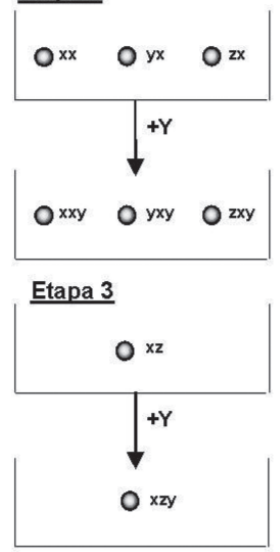

Composto Ativo

Figura 4. Processo de deconvolução para uma biblioteca de 27 elementos

normalmente aplicada na síntese de fármacos; apenas recentemente uma adaptação desta técnica foi utilizada na síntese de materiais inorgânicos sólidos ${ }^{19}$. Entretanto, mesmo na área de fármacos, a síntese paralela vem adquirindo cada vez mais importância, devido à sua menor complexidade.

\section{Síntese via "thin-film vapor deposition"}

A deposição de filmes finos em substratos ${ }^{4,5,20}$ é uma técnica normalmente empregada na indústria de semicondutores. Um sistema de deposição (por ex., "RF-sputtering", deposição eletroquímica, ablação por laser pulsado ou evaporação por feixe de elétrons) é sempre usado em conjunto com técnicas de mascaramento ("masking"), havendo deposição de diferentes substâncias em diferentes áreas de um substrato, de forma sequiencial ou simultânea. O projeto e a seqüência das máscaras define as substâncias a serem depositadas em determinada área do substrato. Através do controle de tempo e velocidade de deposição é possível controlar a composição química exata de cada componente da biblioteca. Em função da pequena barreira de difusão entre as finas camadas depositadas, um tratamento térmico à baixa temperatura permite a obtenção de bibliotecas com um grande número de componentes ${ }^{5}$.

Quando se usa esta abordagem, a eficiência com que se faz a preparação de uma biblioteca de materiais é função da estratégia de mascaramento. Geralmente são utilizados os mascaramentos dos tipos binário ou quaternário, que utilizam máscaras móveis que expõem ou escondem certas áreas do substrato, o que permite variações controladas da composição e/ou espessura dos filmes depositados.

Como pode ser visto na Figura 5, a estratégia de máscaramento binário consiste na utilização de uma ou mais máscaras que protegem metade do substrato, a outra metade fica descoberta permitindo a deposição da substância desejada. A Figura 5 mostra ainda que o deslocamento e a troca de máscaras permite a deposição seqüiencial de diferentes substâncias em regiões diversas do substrato.

Quando se aplica a estratégia de mascaramento quaternário, a principal diferença está na utilização de máscaras que protegem três quartos do substrato, deixando exposto, em cada etapa de de- posição, apenas um quarto. Esta última estratégia é mais utilizada no desenvolvimento de bibliotecas com composições muito variadas, como mostra a Figura 5.
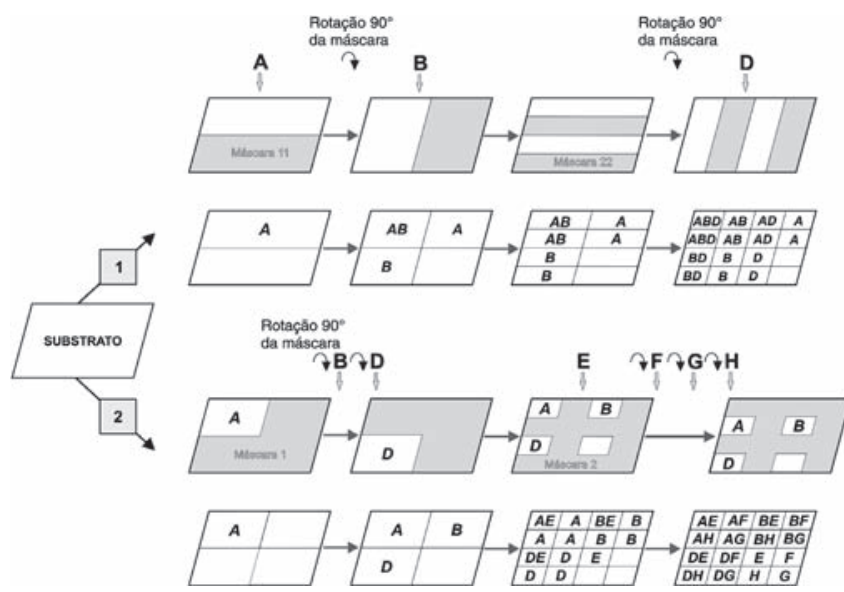

Figura 5. Estratégias de mascaramentos binários e quaternários: 1. mascaramento binário, 2. mascaramento quaternário

\section{Síntese via "ink jet"}

Esta tecnologia utiliza um sistema tipo "ink jet" de cabeças múltiplas (Figura 6), que é usado para realizar microssínteses de bibliotecas de materiais. Nesse sistema, a tinta é substituída por soluções ou por suspensões coloidais dos reagentes. A alta resolução espacial das cabeças múltiplas, associada à precisa dosagem de quantidades, na escala de nano litros, permite a síntese de pequenas quantidades de materiais ${ }^{21}$.

Como pode ser visto na Figura 6, um microcomputador controla um disco piezoelétrico que, por sua vez, determina a abertura dos recipientes que contém os reagentes. O microcomputador controla também uma base móvel do tipo XY, sobre a qual é colocado um suporte com os poços de reação. Gotas de diferentes reagentes podem então ser adicionadas, seqüencialmente, a um mesmo poço, permitindo um controle exato da estequiometria final do material.

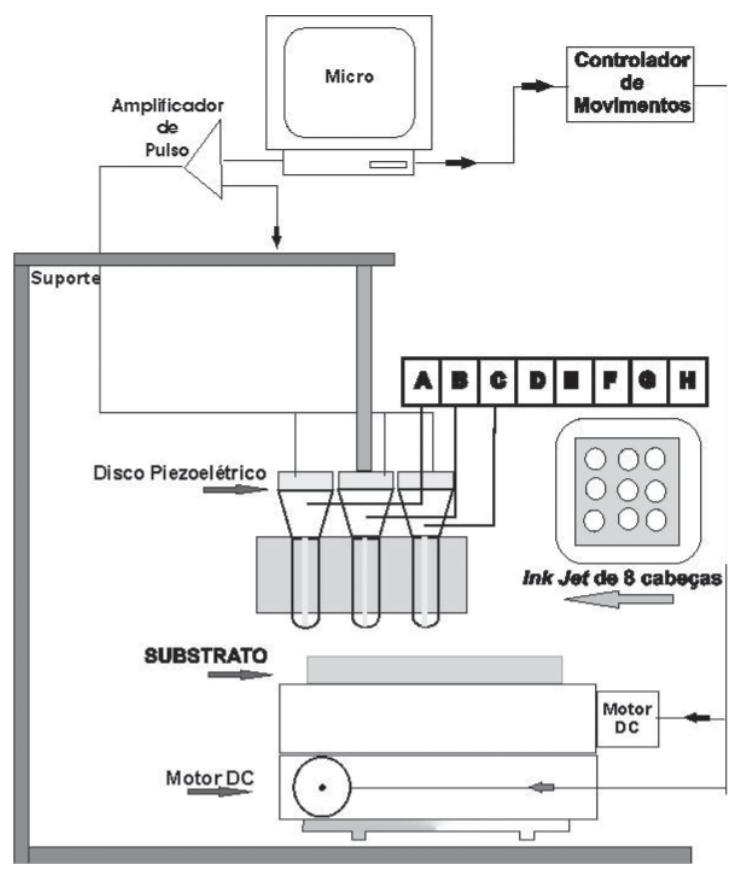

Figura 6. Sistema de injeção "ink jet" 
O sistema tem sido utilizado, com sucesso ${ }^{21}$, para gerar bibliotecas de 100 membros/pol ${ }^{2}$, em curtos espaços de tempo.

\section{AVALIAÇÃO DA BIBLIOTECA POR MDRX}

Como dito anteriormente, a etapa de síntese da química combinatória envolve a preparação de pequenas quantidades de um grande número de materiais com relações estequiométricas e/ou estruturais diversas. Essa etapa é seguida pela avaliação dos componentes da biblioteca, onde é feita uma verificação das propriedades de interesse. A avaliação pode ser realizada individualmente em cada componente, quando técnicas pontuais como a difração de raios $\mathrm{X}$ (DRX) são utilizadas ${ }^{17}$, ou simultaneamente na biblioteca toda, no caso da utilização de técnicas com resolução espacial como, por ex., a termografia com câmeras de vídeo no infravermelho ${ }^{10,11}$.

O grande desafio está na avaliação de materiais e catalisadores, uma vez que há necessidade de se dispor de técnicas rápidas para testar e avaliar variações na composição, estrutura e propriedades dos materiais de uma biblioteca. A maioria dos trabalhos atuais, nas áreas de materiais ou catálise combinatória, está mais focada no desenvolvimento das técnicas de avaliação que na descoberta de novos catalisadores ou materiais.

A técnica de DRX é bastante atraente por não ser destrutiva, ser capaz de fazer uma coleta de dados rápida, ter capacidade de analisar camadas não superficiais devido à penetração dos raios $\mathrm{X}$ e de fornecer várias informações úteis, como as existentes em um difratograma.

Nos últimos anos, a DRX sofreu avanços no sentido de adequála à caracterização de amostras oriundas de sínteses combinatórias, onde há necessidade de se analisar amostras muito pequenas no menor tempo possível. Por exemplo, podem ser realizadas em um único experimento 1.000 combinações de sínteses de zeólitas, possuindo cada uma no máximo $10 \mathrm{mg}$ de material ${ }^{17}$.

$\mathrm{O}$ conceito de microdifração de raios X (MDRX) foi introduzi$\mathrm{do}^{22}$ para os casos nos quais a área a ser analisada é menor que $1 \mathrm{~mm}^{2}$. Para tornar possível a MDRX, o principal obstáculo vencido foi o da divergência dos raios- $X$, portanto, os maiores desenvolvimentos realizados foram na parte ótica, como detalhado a seguir ${ }^{22-27}$.

\section{Desenvolvimentos óticos}

Em trabalhos apresentados na Conferência de Raios X de Denver, em 1998 ${ }^{23}$, começaram a ser discutidas utilizações de canais capilares ocos para a produção de micro feixes de raios $\mathrm{X}$. Esses capilares são de vidro e utilizam o fenômeno de reflexão total, pelas suas paredes internas que são lisas, o que permite a concentração e o direcionamento dos raios X. Como resultado, é produzido um feixe intenso, com diâmetro de micrômetros, o que torna possível a obtenção de um microfoco com as dimensões necessárias para aplicação em MDRX. Nos trabalhos apresentados posteriormente, sobre o assunto, foram idealizadas e testadas várias óticas baseadas nesses capilares ${ }^{24}$. As óticas disponíveis hoje podem ser de dois tipos: monocapilar ou policapilar monolítico.

A Figura 7 apresenta a ótica tipo monocapilar, que é constituída por um canal capilar único, reto ou cônico, com capacidade de guiar e focar, por meio de reflexões múltiplas, os raios $\mathrm{X}$ provenientes de uma fonte pontual ou de uma fonte síncroton, formando um feixe bastante intenso. Os capilares retos fornecem um feixe de alguns micrômetros de diâmetro, enquanto os cônicos fornecem feixes menores, com diâmetros que podem chegar à ordem de submicrômetros ${ }^{24-26}$.

Os monocapilares cônicos concentram os raios $\mathrm{X}$ provenientes de vários tamanhos de fontes, fornecendo pontos ("spots") com fo-

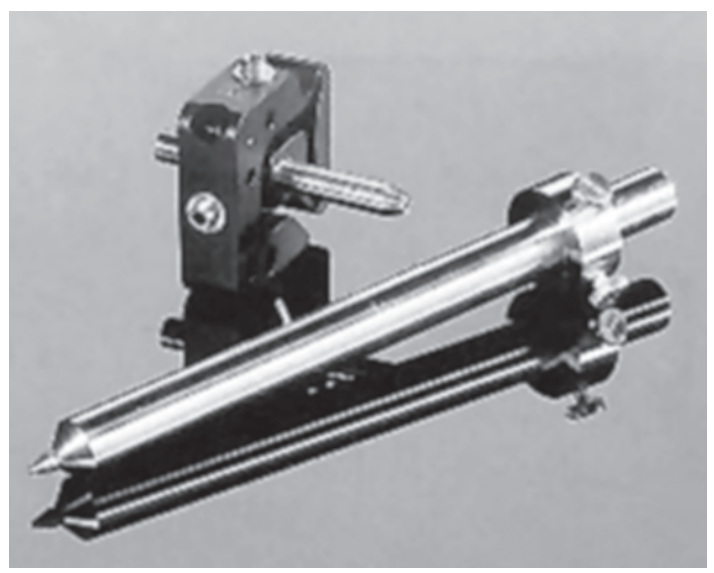

Figura 7. Ótica monocapilar e suporte

cos que geralmente variam de $1 \mathrm{a} 100 \mu \mathrm{m}$, dependendo da fonte e da aplicação. A faixa de divergência desses feixes focados é da ordem de $0,5^{\circ}$ para fótons de $\mathrm{Cu} \mathrm{K}_{\alpha}(8 \mathrm{keV})$. $\mathrm{O}$ ganho no fluxo gerado pode ser de até 100 vezes daquele obtido pelos "pinholes" comuns.

Esses feixes, apesar de serem significantemente mais intensos, ainda apresentam limitações. O feixe de saída diverge, depois de deixar a ótica, obrigando que a amostra seja colocada muito próxima a esta saída, de modo a utilizar a intensidade do feixe de forma mais eficiente. Para resolver esta limitação, surgiram as óticas constituídas por policapilares, conhecidos como policapilares monolíticos ${ }^{24-26}$.

Nas óticas policapilares monolíticas, Figuras 8 a e 8b, centenas de milhares de canais capilares são fundidos juntos, na forma desejável. Sob o ponto de vista da geometria, o diâmetro de cada capilar deve ser menor que o seu raio de curvatura, para garantir que os raios $\mathrm{X}$ incidam nas paredes em ângulos menores que $\mathrm{o}$ ângulo crítico para reflexão total. Mais especificamente, tem-se que $\mathrm{R}>2 \mathrm{~d} / \theta_{\mathrm{c}}^{2}$, onde $\mathrm{R}$ é o raio de curvatura do capilar, $\mathrm{d}$ o diâmetro do capilar e $\theta_{c}$ o ângulo crítico para reflexão total. Para vidros de sílica, comumente utilizados devido ao polimento das paredes e à facilidade de moldagem, o $\theta_{c}$ é de aproximadamente 30/E mrad, onde $\mathrm{E}$ é a energia, em $\mathrm{keV}$, dos raios $\mathrm{X}$ utilizados ${ }^{25,26}$.

Na MDRX podem ser utilizadas a ótica focalizadora ou a ótica colimadora $^{25}$, Figura 9. A característica mais importante da ótica

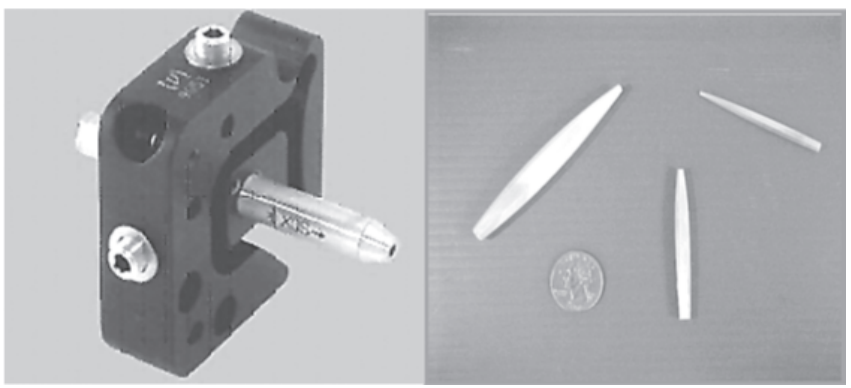

Figura 8a. Ótica policapilar monolítica (parte externa)

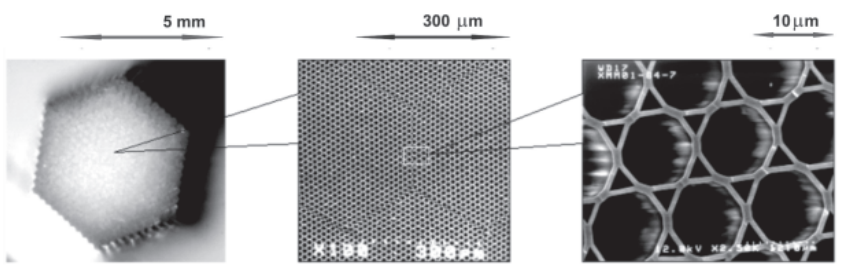

Figura 8 b. Ótica policapilar monolítica (parte interna) 
focalizadora é a existência de dois pontos focais, um na entrada e outro na saída da ótica; a concavidade de todos os canais individuais é dirigida para esses dois pontos. Quando uma fonte de raios X é colocada em um dos pontos de foco, um ângulo sólido grande $\left(\sim 10^{\circ}\right)$ pode ser coletado pela ótica e concentrado no outro foco. Para o caso da ótica colimadora, os raios-X coletados são transformados em um feixe intenso e paralelo. Outra vantagem destes dois tipos de ótica, é o maior espaço de trabalho disponibilizado no lado da amostra. $\mathrm{O}$ diâmetro do feixe produzido pode alcançar até $40 \mu \mathrm{m}$, sendo dependente da divergência do feixe nos capilares individuais e da distância focal da ótica. As óticas policapilares monolíticas comercializadas são cilíndricas, possuindo $8 \mathrm{~mm}$ de diâmetro e $70 \mathrm{~mm}$ de comprimento.

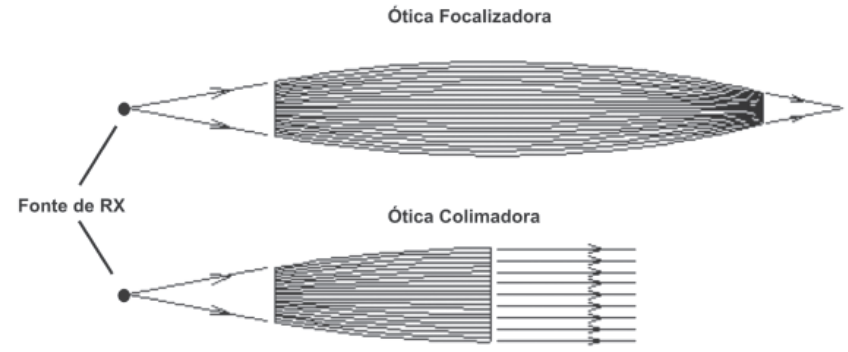

Figura 9. Esquema das óticas policapilares focalizadora e colimadora

Uma outra forma de produção de microfeixe, para MDRX, consiste na introdução de um espelho de Göbel no feixe de raios $X^{26,27}$. Esses espelhos são do tipo multicamadas, sendo refletores artificiais que são projetados para produzir difração de Bragg. Possuem em sua superfície de 100 a 200 camadas nanométricas, alternadas, de dois elementos químicos diferentes. Eles são produzidos através da deposição de camadas, com precisão da ordem de picômetros, utilizando-se as técnicas de evaporação por feixe de elétrons ou de deposição por laser pulsado, sendo que esta última é realizada em pouquíssimos laboratórios do mundo.

As configurações desta ótica podem ser simples ("Single Göbel Mirror", SGM) ou pareadas ("Twin Göbel Mirror", TGM), conforme mostrado nas Figuras 10a e 10b, respectivamente. Esses espelhos coletam os feixes provenientes de tubos convencionais de raios $\mathrm{X}$, que podem apresentar focos em linha ou em ponto, formando na saída um feixe intenso e paralelo com divergência muito baixa. Oferecem como vantagens um aumento substancial na intensidade do feixe primário que incide na amostra, a possibilidade de alinhamento fácil e rápido dessa amostra, uma supressão efetiva das radiações não monocromáticas (contínuo, fluorescência, dentre outras) e um aumento na relação sinal/ruído. Além disso, é possível a obtenção de feixes de até $50 \mu \mathrm{m}$ de seção transversal ${ }^{26,27}$.

As amostras produzidas pela química combinatória devem ser analisadas no menor espaço de tempo possível. Assim, para tornar possível a aplicação da técnica de MDRX, na análise dos compo-

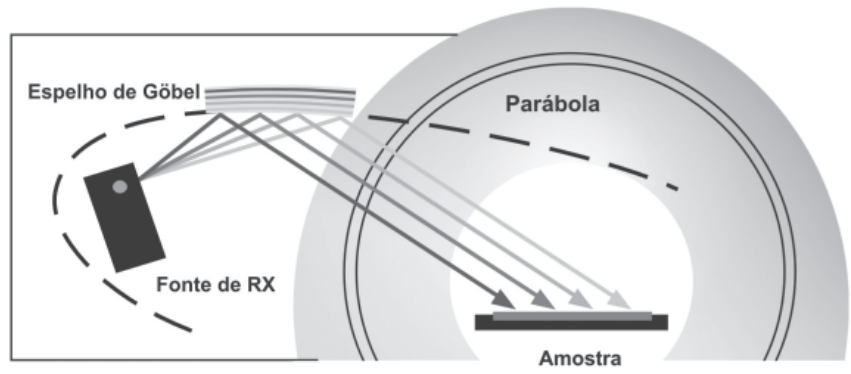

Figura 10a. Espelho de Göbel em configuração simples

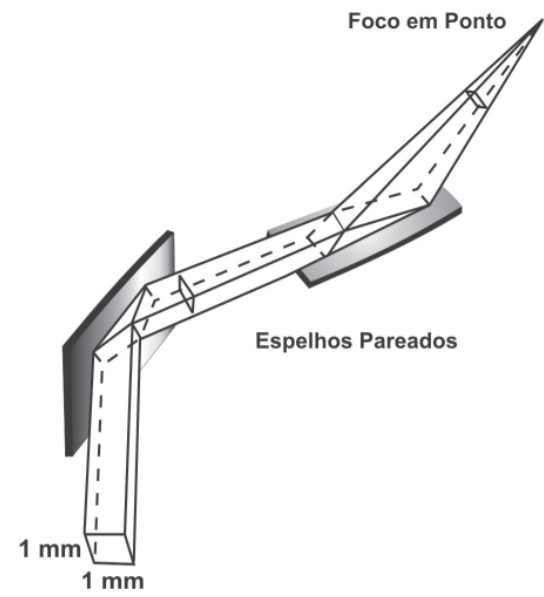

Figura 10b. Espelho de Göbel em configuração pareada

nentes de uma biblioteca combinatória, houve ainda a necessidade de se adequar os porta-amostras e os detectores.

\section{Porta-amostra}

Ao invés de se utilizar os porta-amostras comuns, são utilizados suportes montados sobre estágios que possuem movimentos $\mathrm{XYZ}$ altamente precisos (Figura 11). As sínteses podem ser feitas diretamente nestes suportes, conhecidos como estágios XYZ. As amostras contidas nestas placas são posicionadas e alinhadas no feixe de raios $\mathrm{X}$ com utilização de um sistema composto por um laser e um microscópio/vídeo (Figura 12). O feixe de laser e o eixo ótico do microscópio/vídeo devem se cruzar sobre a amostra, que deve estar posicionada no centro do instrumento. A posição da amostra pode, então, ser determinada e ajustada pela localização do "spot" do laser. O vídeo do microscópio registra a imagem e a posição de cada célula da biblioteca, durante a coleta de dados.

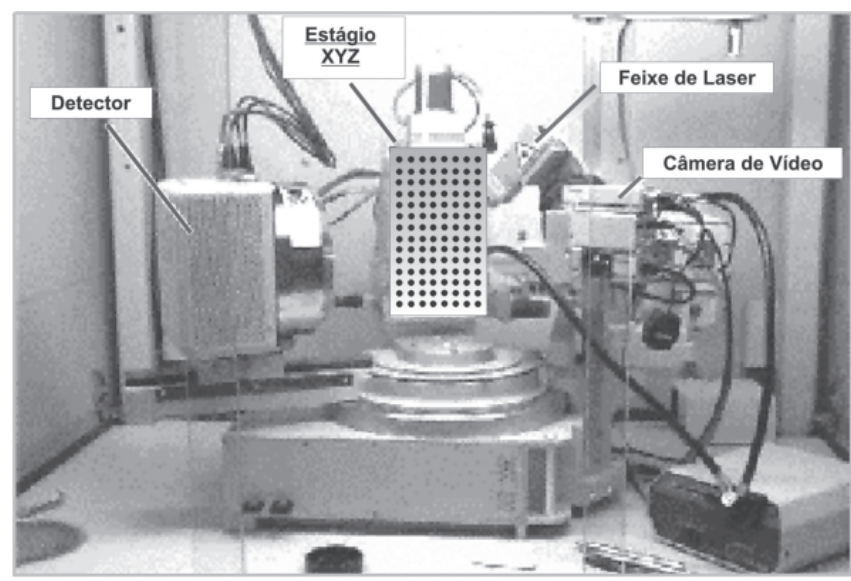

Figura 11. Porta-amostra com estágio $X Y Z$

\section{Detector}

O cintilômetro, normalmente utilizado na DRX, não é o detector mais vantajoso para utilização em análises de amostras provenientes da química combinatória, uma vez que nesse detector não há simultaneidade da coleta de dados, havendo a necessidade de se realizar uma varredura no ângulo de difração, o que é feito por um goniômetro. O mais adequado é um detector bidimensional sensível à posição ("position-sensitive detector", PSD). O PSD é essen- 
cialmente um detector proporcional a gás, no qual as eletrônicas de coleta dos elétrons e de geração dos pulsos estão ligadas aos dois terminais do arame que atua como ânodo. O ânodo é um condutor pobre, com o objetivo de tornar mais lenta a passagem dos elétrons. A velocidade de evolução do pulso ("the rise time"), em cada terminal do arame, é relacionada à posição do arame na qual a ionização se originou.

Os detectores PSD têm sido utilizados desde meados dos anos 1970, mas só recentemente suas aplicações vêm crescendo. Eles são imprescindíveis no caso da caracterização de amostras preparadas por química combinatória, devido à sua capacidade de registrar dados de uma larga faixa de ângulos, simultaneamente. Os desenvolvimentos eletrônicos possibilitaram a obtenção de um difratograma em alguns minutos. A resolução angular efetiva é de alguns centésimos de graus $2 \theta$, suficiente para a maioria das necessidades da química combinatória ${ }^{28,29}$.

A Figura 12 mostra um esquema de aparelho de micro difração de raios $\mathrm{X}$, com o colimador óptico, o estágio $\mathrm{XYZ}$, a fonte de laser para focalização da amostra, o microscópio e o detector sensível à posição.

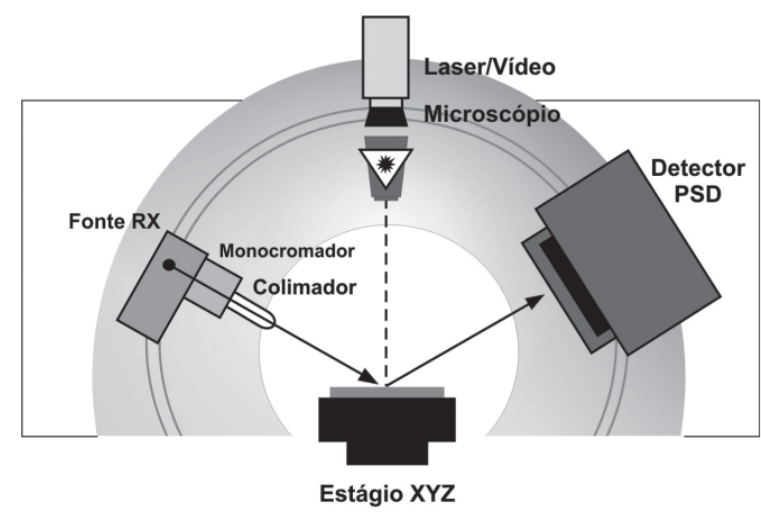

Figura 12. Esquema de um microdifratômetro de raios $X-$ Bruker

\section{CONCLUSÃO}

Métodos para a química combinatória aplicada a materiais estão em desenvolvimento muito rápido e estratégias experimentais adequadas a cada metodologia estão aparecendo também rapidamente $\mathrm{s}^{5,11,15-19}$. Uma das grandes limitações para a aplicação desta abordagem, de maneira mais abrangente, consiste no desenvolvimento de técnicas analíticas automatizadas que permitam obter, de forma rápida e confiável, informações relevantes sobre o sistema em estudo.

$\mathrm{Na}$ área de sólidos inorgânicos, a difração de raios-X é uma das técnicas analíticas mais abrangentes. Entretanto, para sua aplicação em química combinatória, de maneira satisfatória, é necessário que se utilize a MDRX; o que implica na compra de equipamentos que são cerca de duas a três vezes mais caros que os equipamentos de DRX convencionais. Portanto, para justificar sua utilização, devem ser consideradas as seguintes vantagens da abordagem combinatória:

permite liberar o químico para atividades de maior demanda intelectual;

possibilita uma grande diminuição no impacto ambiental do trabalho de pesquisa, uma vez que se utilizam micro amostras; torna possível a realização de um grande número de experimentos em um espaço de tempo relativamente curto, o que diminui sensivelmente o custo individual dos experimentos e utiliza quantidades menores de reagentes, o que também diminui os custos.
Finalizando, pode-se concluir que a combinação da MDRX com as metodologias de síntese, descritas neste artigo, possibilitará que o preparo e a análise de um grande número de amostras sejam feitos em um único dia, tornando a química combinatória capaz de ter um impacto altamente significativo na descoberta e otimização de materiais.

\section{REFERÊNCIAS}

1. Hsieh-Wilson, L. C.; Xiang, X. D.; Schultz, P. G.; Acc. Chem. Res. 1996, 29, 164.

2. Czarnik, A. W.; Acc. Chem. Res. 1996, 29, 112.

3. Gennari, F.; Seneci, P.; Miertus, S.; Catal. Rev. Sci. Eng. 2000, 42, 385.

4. Xiang, X. D.; Sun, X.; Briceno, G.; Lou, Y.; Wang, K. -A.; Cahng, H.; Wallace-Freedman, W. G.; Chen, S.-W.; Schultz, P. G.; Science 1995, 268, 1738 .

5. Cawse, J. N.; Acc. Chem. Res. 2001, 34, 213.

6. Terret, N. K.; Combinatorial Chemistry, Oxford University Press: Oxford, 1998.

7. Fenniri, H.; Curr. Med. Chem. 1996, 3, 343; Martin, E. J.; Critchlow, R. E.; J. Comb. Chem. 1999, 1, 32.

8. Lam, K. S.; Lebl, M.; Krchnak, V.; Chem. Rev. 1997, 97, 411; Leitão, A.; Montanari, C. A.; Donnici, C. L.; Quim. Nova 2000, 23, 178; Dias, R. L. A.; Corrêa, A. G.; Quim. Nova 2001, 24, 236.

9. Murer, P.; Lewandowski, K.; Svec, F.; Fréchet, J. M. J.; Chem. Commun. 1998, 2559; Boussie, T. R.; Coufard, C.; Turner, H.; Murphy, V.; Powerds, T.; Angew. Chem., Int. Ed. 1998, 37, 3472.

10. Bein, T.; Angew. Chem., Int. Ed. 1999, 38, 323; Hagemeyer, A.; Jandeleit, B.; Liu, Y.; Poojary, D. M.; Turner, H. W.; Volpe Jr., A. F.; Weinberg, W. H.; Appl. Catal., A 2001, 221, 23; Scheidtmann, J.; Weiß, P. A.; Maier, W. F.; Appl. Catal., A 2001, 222, 79.

11. Jandeleit, B.; Schaefer, D. J.; Powers, T. S.; Turner, H. W.; Weinberg, W. H.; Angew. Chem., Int. Ed. 1999, 38, 2494; Pescarmona, P. P.; van de Waal, J. C.; Maxwell, I. E.; Maschmeyer, T.; Catal. Lett. 1999, 63, 1; Lettmann, C.; Hinrichs, H.; Maier, W. F.; Angew. Chem., Int. Ed. 2001, 40, 3160; Urschey, J.; Kühnle, A.; Maier, W. F.; Appl. Catal., A 2003, 252, 91.

12. Shimizu, K. D.; Snapper, M. L.; Hoyveda, A. H.; Chem. Eur. J. 1998, 4, 1885; Hoyveda, A. H.; Chem. Biol. 1998, 5, R187; Kagan, H. B.; J. Organomet. Chem. 1998, 567, 3.

13. Persidis, A.; Chem. Ind. (London) 1998, Oct., 782; Bellavance, L. L.; Chem. Eng. (New York) 1999, Sep., 76.

14. Maier, W. F.; Angew. Chem., Int. Ed. 1999, 38, 1216; Senkan, S.; Angew. Chem., Int. Ed. 2001, 40, 312.

15. Holzwarth, A.; Denton, P.; Zanthoff, H.; Mirodatos, C.; Catal. Today 2001, 67, 309; Koinuma, H.; Appl. Surf. Sci. 2002, 189, 179; Smotkin, E. S.; Díaz-Morales, R. R.; Annu. Rev. Mater. Res. 2003, 33, 557; Kirsten, G.; Maier, W. F.; Appl. Surf. Sci. 2004, 223, 87.

16. Newsam, J. M.; Bein, T.; Klein, J.; Maier, W. F.; Stichert, W.; Microporous Mesoporous Mater. 2001, 48, 355; Schuth, F.; Busch, O.; Hoffmann, C.; Johann, T.; Kiener, C.; Demuth, D.; Klein, J.; Schunk, S.; Strehlau, W.; Zech, T.; Top. Catal. 2002, 21, 55; Caruthers, J. M.; Lauterbach, J. A.; Thomson, K. T.; Venkatasubramanian, V.; Snively, C. M.; Bhan, A.; Katare, S.; Oskarsdottir, G.; J. Catal. 2003, 216, 98.

17. Akporiaye, D. E.; Dahl, I. M.; Karlsson, A.; Wendelbo, R.; Angew. Chem., Int. Ed. 1998, 37, 609; Klein, J.; Lehmann, C. W.; Schmidt, H. W.; Maier, W. F.; Angew. Chem., Int. Ed. 1998, 37, 3369; Lai, R.; Kang, B. S.; Gavalas, R.; Angew. Chem., Int. Ed. 2001, 40, 408; Yanase, I.; Ohtaki, T.; Watanabe, M.; Solid State Ionics 2002, 151, 189; Yanase, I.; Ohtaki, T.; Watanabe, M.; Solid State Ionics 2002, 154-155, 419; Yanase, I.; Ohtaki, T.; Watanabe, M.; Appl. Surf. Sci. 2002, 189, 292.

18. Moates, F. C.; Somani, M.; Annamalai, J.; Richardson, J. T.; Luss, D.; Willson, R. C.; Ind. Eng. Chem. Res. 1996, 35, 4801; Claus, P.; Hönicke, D.; Zech, T.; Catal. Today 2001, 67, 319; Morris, N. D.; Mallouk, T. E.; J. Am. Chem. Soc. 2002, 124, 11114.

19. Sun, Y.; Chan, B. C.; Ramnarayanan, R.; Leventry, W. M.; Mallouk, T. E.; Bare, S. R.; Willis, R. R.; J. Comb. Chem. 2002, 4, 569.

20. Danielson, E.; Golden, J. H.; McFarland, E. W.; Reaves, C. M.; Weinberg, W. H.; Wu, X. D.; Nature 1997, 389, 944; Pirrung, M. C.; Chem. Rev. 1997, 97, 473; Johson, D. A.; Solid State Mater. 1998, 3, 159; MacFarland, E. W.; Weinberg W. H.; Trends Biotechnol. 1999, 17, 107; Koinuma, H.; Aiyer, H. M.; Matsumoto, Y.; Sci. Technol. Adv. Mater. 2000, $1,1$.

21. Sun, X. D.; Wang, K. A.; Yoo, W.; Wallace-Freedman, W. G.; Gao, C.; Xiang, X. D.; Schultz, P. G.; Adv. Mater. 1997, 9, 1046; Reddington, E.; Sapienza, A.; Gurau, B.; Wiswananthan, R.; Satangapani, S.; Smotkin, E. S.; Mallouk, T. E.; Science 1998, 280, 1735; Tay, B. Y.; Edirisinghe, M. J.; J. Mater. Res. 2001, 16, 373; Evans, J. R. G.; Edirisinghe, M. J.; Coveney, P. V.; Eames, J.; J. Eur. Ceram. Soc. 2001, 21, 2291. 
22. Fransen, M. J.; Vasterink, J. H. A.; Nijenhuis, J.; Adv. X-Ray Anal. 2001 $44,284$.

23. Kanngieber, B.; Beckhoff, B.; Malzer, W.; Arkadiev, V. A.; Bzhaumikhov, A. A.; Gorny, H.-E.; Adv. X-Ray Anal. 1998, 40, 526; Yan, Y.; Adv. X-Ray Anal. 1998, 40, 542.

24. He, B. B.; Preckwinkel, U.; Smith, K. L.; Adv. X-Ray Anal. 1999, 43, 439; He, B. B.; Anzelmo, J.; LaPuma, P.; Preckwinkel, U.; Smith, K.; Adv. XRay Anal. 2001, 44, 1; Ignatiev, K.; Rek, Z. U.; Stock, S. R.; Adv. X-Ray Anal. 2001, 44, 56; Havrilla, G. J.; Mann, G.; Warner, B.; Adv. X-Ray Anal. 2001, 44, 74; Gubarev, M.; Ciszak, E.; Ponomarev, I.; Gibson, W.; Joy, M.; Adv. X-Ray Anal. 2001, 44, 278; Chang, W.; Kerner, J.; Franco, E.; Adv. X-
Ray Anal. 2001, 44, 325; Soejima, H.; Narusawa, T.; Adv. X-Ray Anal. 2001, 44, 320; Reyes-Mena, A.; Cornaby, S.; Pew, H. K.; Moody, P. W.; Hughes, T.; Stradling, A.; Knight, L. V.; Adv. X-Ray Anal. 2001, 44, 343.

25. http://www.xos.com, acessada em Novembro 2003.

26. Hildebrandt, G.; Bradaczec, H.; The Rigaku J. 2000, 17, 13.

27. Schuster, M.; Göbel, H.; J. Phys. D: Appl. Phys. 1995, 28, A270.

28. Jenkins, R.; Gould, R. W.; Gedcke, D.; Quantitative X-ray Spectrometry, Marcel Dekker, Inc.: USA, 1995, p. 484.

29. Jenkins, R.; Snyder, R.L.; Introduction to X-ray Powder Diffractometry, John Wiley \& Sons, Inc.: USA, 1996, p. 403 